\title{
Grain Size Analysis in Lithium Aluminate Ceramics
}

\author{
Bradley R. Johnson ${ }^{1}$, Nathan Canfield ${ }^{1}$, Jarrod V. Crum ${ }^{1}$ and Benjamin McCarthy \\ 1. Pacific Northwest National Laboratory, Energy and Environment Directorate, Richland, WA.
}

Grain size is an important structural feature of ceramic and metallic specimens. For a given material and fabrication process, variations in average grain size and/or grain size distribution can be correlated to variations in mechanical or electrical properties. Thus, grain size measurements are often used as a quality control metric to evaluate a fabrication process without having to do extensive mechanical or electrical testing for each lot.

A traditional method for measuring grain size is to manually count grain boundary intercepts from a micrograph with a calibrated scale bar. ASTM E112 [1] describes several standardized approaches for determining grain size in this manner. A newer approach based on electron backscattered diffraction (EBSD), was introduced in ASTM E2627 [2]. This was developed to establish a standardized way to use EBSD patterns as a means to distinguish between grains based on their crystallographic orientation. This can have several advantages for suitably polished specimens. For example, it can be easier to distinguish between different grains based on their crystallographic orientation via EBSD, than can be determined by visually identifying grain boundaries. Additionally, EBSD mapping can produce a more quantitative analysis of grain data, such as a histogram that shows the distribution of grain sizes, which is not possible with manual grain boundary intercept counting methods. And, EBSD is able to distinguish between multiple phases and void fraction in a sample, whereas the intercept counting method has no method to deal with these. Success with either approach requires high quality metallographic sample preparation.

Lithium aluminate ceramics $\left(\mathrm{LiAlO}_{2}\right)$ have proven to be a problematic material for grain size analysis. There are several issues that have to be addressed. First, the material is water-sensitive, so it must be prepared using a non-aqueous polishing protocol. Second, the material is multi-phase: contaminants remain in the specimen from the milling the process (e.g. $\mathrm{ZrO}_{2}$ fines from ball milling); pores and hard agglomerates exist due to incomplete compaction and non-uniform densification of the initial powders; and alternate lithium aluminate phases (e.g. $\left.\mathrm{LiAl}_{5} \mathrm{O}_{8}\right)$ are present due to small variations in stoichiometry. Since both the $\mathrm{ZrO}_{2}$ and $\mathrm{LiAl}_{5} \mathrm{O}_{8}$ phases are harder than the $\mathrm{LiAlO}_{2}$ matrix, they polish slower, and at the end of the polishing process, the harder phases appear as protrusions on the specimen surface.

An optimized polishing process was developed using $\mathrm{SiC}$ papers and progressing to woven polishing cloths with diamond suspensions at $9 \mu \mathrm{m}$, and $3 \mu \mathrm{m}$. Final polishing was accomplished using a vibratory polisher, a low-nap polishing pad, and non-aqueous colloidal $\mathrm{SiO}_{2}$. This approach was able to produce superior surface finishes that were adequate for collecting EBSD maps.

Imaging conditions for grain size analysis were evaluated using various accelerating voltages, beam currents, detectors, vacuum levels and working distances. Example micrographs are shown in Figure 1. Sputter coating the specimens with Au or Pt provided a conductive coating for high vacuum imaging, but it eliminated the orientation grain contrast in the micrographs. Orientation contrast images could be obtained under high vac conditions with a 5-10 nm carbon coating. However, the carbon coating slightly muted the orientation contrast. Optimum grain orientation contrast images were obtained with un-coated specimens under low vacuum conditions, using a backscatter electron detector at $5 \mathrm{kV}, 1-4 \mathrm{nA}, \mathrm{WD}=4-$ 
$5 \mathrm{~mm}$, and 30Pa. Micrographs collected under these conditions contained superior gray scale contrast between grains based on their crystallographic orientation (Figure 2). Grain size analysis could be successfully performed on these micrographs by counting grain boundary intercepts using the three-circle intercept method specified in ASTM E112.

The ASTM E2627 method for conducting grain size analysis is advantageous due to the superior grain statistics and automated measurement approach. However, in these multi-phase ceramics, the harder phases protruded above the plane of the specimen. Due to the high tilt angle inherent in EBSD analysis, the protrusions shadowed the portion of the specimen below it, thereby interfering with accurately collecting patterns from those grains. The harder phases also interfered with the quality of the surface finish of the matrix in the vicinity of the protrusion, thereby degrading EBSD pattern quality around those phases. These issues resulted in errors for the EBSD analysis of grain size that the manual method did not have. Consequently, sample preparation again becomes the limiting factor for accurate measurements. The conclusion was that manual methods that rely on operator judgement to discriminate between phases remained a viable approach to measuring grain size for stringent quality control measurements.

References:

[1] ASTM Standard E112-96, ASTM International, West Conshohocken, PA (2004).

[2] ASTM Standard E2627-13, ASTM International, West Conshohocken, PA (2013).
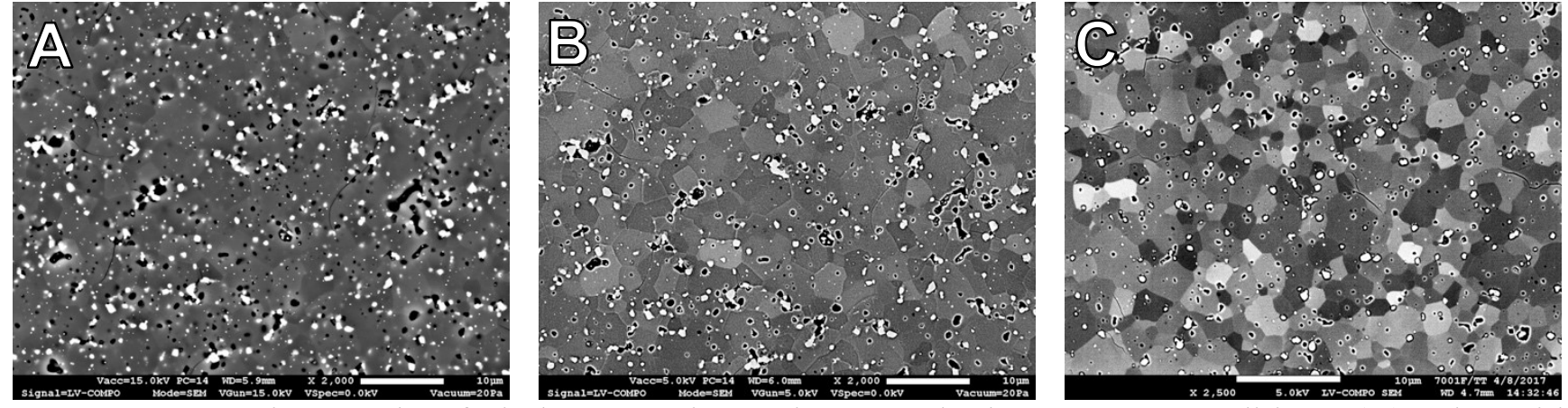

Figure 1. BSE Micrographs of $\mathrm{LiAlO}_{2}$ ceramic specimens under low vacuum conditions. (A) $15 \mathrm{kV}$ with $\mathrm{C}$ coating; (B) $5 \mathrm{kV}$ with $\mathrm{C}$ coating; and (C) $5 \mathrm{kV}$ with no coating.
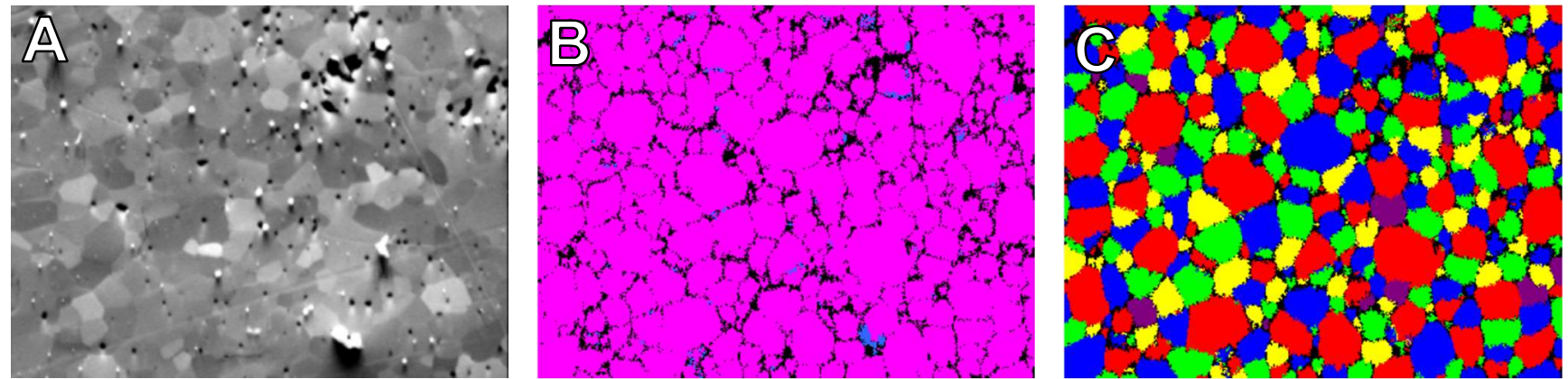

Figure 2. EBSD micrographs of $\mathrm{LiAlO}_{2}$ : (A) forscatter , (B) phase map (magenta $=\mathrm{LiAlO}_{2}$, blue $=$ $\mathrm{LiAl}_{5} \mathrm{O}_{8}$ ), and (C) grain size. 